\title{
La aspirina reduce el riesgo de recurrencia de enfermedad tromboembólica venosa idiopática
}

Aspirin reduces the risk of recurrence of idiopathic venous thromboembolism

Becattini C y col. N Engl J Med 2012;366:1959-67.

\section{Objetivos}

Comparar la eficacia de $100 \mathrm{mg}$ de aspirina contra placebo para prevenir la recurrencia de enfermedad tromboembólica venosa (ETV) en pacientes que completaron el tratamiento con antagonistas de vitamina K (AVK) por ETV idiopática.

\section{Diseño, lugar y pacientes}

Ensayo clínico aleatorizado, multicéntrico, doble ciego, realizado en diez centros de Italia y uno de Austria. Participaron 403 pacientes mayores de 18 años que habían cumplido con 6 a 18 meses de tratamiento anticoagulante con AVK por haber presentado un primer episodio sintomático, objetivamente confirmado, de ETV idiopática, tanto trombosis venosa profunda (TVP) proximal como tromboembolismo de pulmón (TEP). Se consideró al episodio idiopático cuando en el momento del diagnóstico no se pudo constatar ningún factor predisponente.

\section{Intervención}

Los pacientes elegibles fueron asignados de forma aleatorizada a dos grupos. El primero recibió $100 \mathrm{mg}$ diarios de aspirina, y el segundo placebo; ambos durante dos años. La aleatorización se realizó a las dos semanas de suspender el tratamiento con AVK. Fueron evaluados cada tres meses el primer año, y cada seis meses luego, y/o en caso de presentar síntomas compatibles con recurrencia de ETV o sangrado. Los pacientes y los miembros del comité que definió la presencia o no de eventos estaban ciegos con respecto al tratamiento.

\begin{abstract}
Medición de resultados principales
El resultado primario de eficacia fue la confirmación objetiva de una recurrencia sintomática de ETV (TVP o TEP); y el resultado primario de seguridad fueron los sangrados mayores. Los resultados secundarios fueron eventos vasculares y muerte de cualquier causa. El análisis se hizo teniendo en cuenta la intención de tratar (se incluía si el paciente había recibido al menos una dosis de tratamiento).
\end{abstract}

\section{Resultados Principales}

Entre 2004 y 2010 se incluyeron 403 pacientes: 205 recibieron AAS; 197, placebo y uno no recibió nada. El período promedio de participación fue de 24 meses en ambos grupos. La recurrencia global fue del $8,6 \% ; 6,6 \%$ en el grupo aspirina y $11,2 \%$ en el placebo (HR 0,58; IC95\% 0,36 a 0,93; p 0,02). No hubo relación con el tiempo de anticoagulación recibido previamente. Hubo un sangrado mayor y un TEP fatal en cada grupo. Las muertes fueron seis y cinco, respectivamente.

\section{Conclusión}

En pacientes con ETV idiopático que completaron el tratamiento con AVK durante 6 a 18 meses, el tratamiento con AAS $100 \mathrm{mg}$ redujo un $40 \%$ el riesgo relativo de recurrencia de ETV, sin aumentar el riesgo de sangrado.

Fuente de financiamiento: Bayer Health Care dio una beca para el estudio y abasteció los comprimidos de AAS y placebo.

\section{Comentario}

El riesgo de recurrencia en la ETV idiopática es elevado, aproximadamente $20 \%$ a los dos años de suspendido el tratamiento con $\mathrm{AVK}^{1-4}$ (habitualmente de 6 a 18 meses de duración). La idea de prolongar la anticoagulación para reducir el riesgo de recurrencia parece tentadora. Sin embargo, siempre se debe balancear con el riesgo de sangrado, que ronda el 2 a $3 \%$ anual con los AVK, cuando se logra un RIN estable (entre 2 y 3). Por otro lado, la AAS ha demostrado reducir el riesgo relativo de eventos de ETV en prevención primaria, entre un 20 y $50 \%{ }^{5,6}$. En los últimos años, se ha sumado una nueva alternativa de prevención en esta ecuación que son los nuevos anticoagulantes orales. Tanto el dabigatrán como rivaroxaban demostraron reducir las recurrencias en este grupo de pacientes en un $80 \%$ en comparación con placebo, sin necesidad de ajuste de dosis ni monitoreo de laboratorio. Sin embar- go, el costo de estas drogas, la falta de un antídoto específico y las contraindicaciones o interacciones, limitan su uso en la práctica habitual.

\section{Conclusiones del comentador}

Debido a la elevada recurrencia de eventos en los pacientes con ETV idiopático después de terminado el tratamiento anticoagulante convencional, y en ausencia de contraindicaciones (alergia, gastritis, hipersensibilidad, etc.), indicar $100 \mathrm{mg}$ diarios de aspirina es una alternativa interesante, eficaz, económica y con bajo riesgo de complicaciones, para reducir el riesgo de eventos de ETV, en comparación con los AVK, los nuevos anticoagulantes orales o no indicar nada.

Fernando Javier Vázquez [ Servicio de Clínica Médica del Hospital Italiano de Buenos Aires. fernando.vazquez@hospitalitaliano.org.ar ]

Vázquez FJ. La aspirina reduce el riesgo de recurrencia de enfermedad tromboembólica venosa idiopática. Evid Act Práct Ambul. 2014, 17(2). AbrJun. 50. Comentado de: Becattini C, Agnelli GC, Schenone A et al. Aspirin for preventing the recurrence of venous thromboembolism. N Engl J Med 2012; 366: 1959-67. PMID: 22621626.

\section{Referencias}

1. Prandoni P, Lensing AW, Cogo A, et al. The long-term clinical course of acute deep venous thrombosis. Ann Intern Med 1996;125:1-7.

2. Agnelli G, Prandoni P, Santamaria MG, et al. Three months versus one year of oral anticoagulant therapy for idiopathic deep venous thrombosis. N Engl J Med 2001;345:165-9. 3. Kearon C, Gent M, Hirsh J, et al. A comparison of three months of anticoagulation with extended anticoagulation for a first episode of idiopathic venous thromboembolism. N Engl J Med 1999:340:901-7.

4. Douketis JD, Gu CS, Schulman S, Ghirarduzzi A, Pengo V, Prandoni P. The risk for fatal pulmonary embolism after discontinuing anticoagulant therapy for venous thromboembolism. Ann Intern Med 2007;147:766-74.

5. Antiplatelet Trialists' Collaboration. Collaborative overview of randomised trials of antiplatelet therapy III. Reduction in venous thrombosis and pulmonary embolism by antiplatelet prophylaxis among surgical and medical patients. BMJ 1994;308:235-46.

6. Prevention of pulmonary embolism and deep vein thrombosis with low dose aspirin: Pulmonary Embolism Prevention (PEP) trial. Lancet 2000;355:1295-302. 